\title{
Genomic analysis of early SARS-CoV-2 breakthrough infections from the state of Kerala suggest preponderance of variants of concern
}

Vettakkara Kandy Muhammed Niyas ${ }^{1, *}$, Afra Shamnath ${ }^{2, *}$, Roshni KJ ${ }^{3}$, Rahul C. Bhoyar ${ }^{2}$, Vimalraj A N ${ }^{5}$, Bani Jolly ${ }^{2,4}$, Anoop Joseph ${ }^{6}$, Vigneshwar Senthivel ${ }^{2,4}$, Rajan J Payyappilly ${ }^{7}$, Mohamed Imran ${ }^{2,4}$, Raman Swathy Vaman $^{8}$, Mercy Rophina ${ }^{2,4}$, Rajendra Pilankatta ${ }^{9}$, Vishu Gupta ${ }^{2,4}$, Lekshmi Balaraman ${ }^{10}$, Arvinden VR ${ }^{2,4}$, Shoba Kurian Pulikottil ${ }^{11}$, Srashti Jyoti Agrawal ${ }^{2,4}$, Pushpa Kizhakkekarammel ${ }^{12}$, Mohit Kumar Divakar ${ }^{2,4}$, Prasanth Viswanathan ${ }^{13}$, Anjali Bajaj ${ }^{2,4}$, Minu Mohan ${ }^{24}$, Rajalakshmi Arjun ${ }^{1}$, Sheeba PM ${ }^{3}$, Sujatha V ${ }^{5}$, Manasi $R^{7}$, Manoj $A T^{8}$, Lathika Valliyott ${ }^{9}$, Alexander VS ${ }^{11}$, Niyas K Pulloor ${ }^{12}$, Saritha Sivadas ${ }^{13}$, Nisha Majeed ${ }^{18}$, Ashalatha $\mathrm{Nair}^{15}$, Anila Mathews ${ }^{3}$, Neethu John ${ }^{23}$, Usha Karunakaran ${ }^{17}$, Ramdas AV ${ }^{8}$, Ranjeet Dungdung ${ }^{9}$, Anitha P.M. ${ }^{12}$, Fairoz Cheriyalingal Parambath ${ }^{19}$, Arun $\mathrm{S}^{20}$, Viji Mohan ${ }^{15}$, V.B Sameer Kumar ${ }^{9}$, Nisha Sadanandan ${ }^{12}$, Priya Chandran ${ }^{14}$, Sarada Devi ${ }^{21}$, Divyamol ${ }^{22}$, Bindhu Vasudevan ${ }^{16}$, Jerrin Maria Jose $^{17}$, Aswathi Kodenchery Somasundaran ${ }^{9}$, Prajit Janardhanan ${ }^{9}$, Vishnu Ramachandran ${ }^{9}$, Sridhar Sivasubbu ${ }^{2,4}$, Vinod Scaria ${ }^{2,4, \#}$

Affiliations:

${ }^{1}$ Department of Infectious Diseases, KIMSHEALTH, Thiruvananthapuram 695029, Kerala, India

${ }^{2}$ CSIR-Institute of Genomics \& Integrative Biology (CSIR-IGIB), Mathura Road, Delhi 110025, India

${ }^{3}$ Department of Microbiology, Government Medical College, Palakkad 678013, India

${ }^{4}$ Academy for Scientific and Innovative Research, Human Resource Development Centre Campus, Ghaziabad 201 002, Uttar Pradesh, India

${ }^{5}$ Department of Microbiology, Government Medical College, Thrissur 680596, Kerala, India

${ }^{6}$ Department of General Medicine, Government Medical College, Ernakulam 683503, Kerala, India

${ }^{7}$ Department of Microbiology, Government Medical College, Kannur 670503, Kerala, India

${ }^{8}$ Department of Health and Family Welfare, Kanhangad 671531, Kerala, India

${ }^{9}$ Department of Biochemistry and Molecular Biology, School of Biological Sciences, Central University of Kerala, Kasargod 671316, Kerala, India

${ }^{10}$ Regional Public Health Laboratory, Pathanamthitta 689641, Kerala, India

${ }^{11}$ Department of Microbiology, Government Medical College, Kottayam 686008, Kerala, India

${ }^{12}$ Department of Microbiology, Government Medical College, Manjeri 676121, Kerala, India

${ }^{13}$ VRDL, Government Medical College, Kozhikode 673008, Kerala, India

${ }^{14}$ Department of Community Medicine, Government Medical College, Kozhikode 673008, Kerala, India

${ }^{15}$ Department of Microbiology, KIMSHEALTH, Thiruvananthapuram 695029, Kerala, India

${ }^{16}$ Department of Community Medicine, Government Medical College, Ernakulam 683503, Kerala, India

${ }^{17}$ Department of Community Medicine, Government Medical College Kannur, Pariyaram 670503, Kerala, India

${ }^{18}$ Department of Microbiology, Government Medical College, Idukki 685602, Kerala, India

${ }^{19}$ Department of Microbiology, Government Medical College, Kozhikode 673008, Kerala, India

${ }^{20}$ Department of Pharmacology, Government Medical College, Idukki 685602, Kerala, India

${ }^{21}$ VRDL, Department of Microbiology, Government Medical College, Trivandrum 695011, Kerala, India

${ }^{22}$ Department of Community Medicine, Government Medical College, Palakkad 678013, Kerala, India

${ }^{23}$ Department of Microbiology, Government Medical College, Ernakulam 683503, Kerala, India 
${ }^{24}$ Department of Community Medicine, Government Medical College, Idukki

\title{
\#Corresponding author:
}

E-mail address: vinods@igib.in (Vinod Scaria)

* contributed equally

\begin{abstract}
The SARS-CoV-2 Variant of Concern, Delta (B.1.617.2) was first reported in December 2020 in India and has spread colossally throughout the globe. Owing to factors like increased transmissibility, immune escape, and virulence, delta variant has been considered as a potential public health threat apart from other variants of concern like alpha, beta and gamma. Kerala was one of the first states in India to enroll in the systematic genomic surveillance. In the present report vaccine breakthrough infections were followed up in 147 patients including 55 healthcare workers who had been vaccinated with ChAdOx1 nCoV19/BBV152 across eleven districts from the state of Kerala. Timeline of samples analysed were from April 2021 till June 2021. Severity of the infections reported in the enrolled patients found to be mildly symptomatic, majorly with only $0.7 \%(n=1)$ of the cohort to be asymptomatic. Genomic analysis of the samples revealed the Delta variant (B.1.617.2) to constitute about $81.6 \%(n=120)$ in the studied cohort. This was followed by the Kappa variant B.1.617.1 (8.35\%, n=9), AY.1 (0.6\%, n= 1) , AY.12 (0.6\%, n= 1), AY.4 (1.2\%, n= 2), AY.9 (1.2\%, n= 2) and Eta variant, B.1.525 $(0.6 \%, n=1) .11$ samples were not assigned any lineage. Evidence from this study suggests preponderance of the Delta variant in the samples analysed.
\end{abstract}

Keywords: SARS-CoV-2, vaccine breakthrough infections, Delta variant.

\section{Introduction}

Vaccine breakthrough infections are infections in fully immunised individuals. While COVID-19 vaccines have been shown to be effective and impart a vigorous immune response ${ }^{1}$, there is emerging evidence to suggest that breakthrough infections occur in a subset of individuals ${ }^{1,2}$. Such breakthrough infections are now reported from across the world 3-5. While a number of studies have consistently suggested that vaccines can significantly reduce the risk for infection, severe disease and death, a number of recent reports suggest emerging variants could cause breakthrough infections ${ }^{6}$. Genomic surveillance of SARS-CoV-2 has been used extensively to identify variants in breakthrough infections, genetic epidemiology as well as aiding public health management ${ }^{7}$.

Tracking the emergence and prevalence of different lineages of SARS-CoV-2 is important to understand how certain mutations change its properties in terms of transmission, pathogenicity and its sensitivity to diagnostic tools, vaccines, and other therapeutics. Currently, 5 lineages of SARS-CoV-2 with potential significance in global public health have been characterized by the WHO as Variants of Concern (VOCs). Of these, the lineage 
B.1.617.2 (VOC Delta) was first detected in India but has since then spread globally to become the predominant lineage in many parts of the world, and has been associated to cause a surge in infections in the regions it is detected. The Delta variant is characterized by key mutations in the Spike protein including L452R, P681R, and T478K which have been previously reported to be associated with increased transmissibility of the virus or in the context of the virus' ability to escape neutralization by antibodies ${ }^{8}$. Concerns have been raised regarding the effectiveness of different vaccines against the Delta variant of SARS-CoV-2. Several recent reports have shown a reduced sensitivity of the variant to the neutralization activity of vaccines. In this study, we describe the genome characterization of vaccine breakthrough infections in 147 patients from Kerala, India, acquired during the time period April-June 2021.

\section{Methodology}

Vaccine breakthrough infections were noted as part of an ongoing genomic surveillance programme in the state of Kerala and according to the institutional ethical guidelines, (IHEC-CSIR-IGIB/IHEC/2020-21/01). Total 147 RT-PCR confirmed cases of SARS-CoV-2 infection were included in the study during the months of April to June 2021. Of 147, 139 patients were fully vaccinated with two doses of Covishield, while 8 were fully vaccinated with two doses of Covaxin. All patients belonged to Kerala, a state in the southern part of India. The mean age of the patients was 39.2 years (Confidence Interval [Cl], 37.12-41.45) (Males ( $n=50)$ Females $(n=99)$. Out of the 147 cases, 99.3\% $(n=146)$ of the cases were symptomatic, $0.7 \%(n=1)$ were asymptomatic and underwent domiciliary care. 55 of the 147 patients were healthcare workers. The interval between first and second dose for all patients was in the range of 28 to 99 days. Symptomatic cases of COVID-19 symptoms were reported in patients with at least 35 days after being fully vaccinated. Clinical details of the patients included in the study are summarized in Supplementary Table 1.

All cases were confirmed using RT-PCR assays on RNA extracted from nasopharyngeal swabs as part of the routine diagnostic workup. Rapid Antigen test was also performed for 16 out of 147 patients after they tested RT-PCR positive for COVID-19. Neutralizing antibody test data was available in 3 patients. Sequencing-ready libraries were prepared from the archived RNA samples using COVIDSeq, an amplicon-based assay (Illumina Inc, CA, USA), in accordance with a recently published protocol ${ }^{9}$. Sequencing was performed on the NovaSeq 6000 platform (Illumina Inc) with 100x2 base pair read-length on SP flow cell. Genomes were assembled using the SARS-CoV-2 reference genome NC_045512.2 ${ }^{10}$. Genome sequences having more than 2000 ambiguous sites were excluded from the phylogenetic analysis. Lineages were assigned to the genome sequences of all samples using pangolin (v3.1.7) ${ }^{11}$. 


\section{Results}

The 147 genome sequences were assembled at a mean genome coverage of 91.5\% (9.4\%-99.99\%) with a mean depth of coverage of 6079.7X (396.5X-12102.5X). Out of 147, 116 breakthrough samples which met the quality cut-offs were used to construct a phylogenetic tree along with 4714 additional genomes sampled from the state of Kerala between December 2020 to July 2021. The resulting tree was annotated and visualized using the $\mathrm{R}$ package ggtree ${ }^{12}$. Figure 1 summarizes the distribution of the lineages in the samples.

The prevalence of the VoCs in the respective districts were compared to understand the relative enrichment for the VoCs of the breakthrough cohort in the present study. Statistical analysis revealed that the VoCs distribution was not significantly distinct among the districts (Fisher's exact test statistic Calicut - 0.4545; Ernakulam - 1; Idukki - 1; Kannur - 0.657; Kasaragod - 1; Kottayam - 1; Malappuram - 1; Palakad - 0.1306; Pathanamthitta - 0.38546; Thrissur - 1; Trivandrum - 1).

The genomic characterization of SARS-CoV-2 breakthrough infections in 147 patients revealed the Delta variant (B.1.617.2) to constitute about 81.6\% ( $n=120)$. Kappa variant B.1.617.1 constituted $8.35 \%(n=9)$ of the lineages. Sub lineages of Delta AY.1 accounted for 0.6\%( $n=1)$, AY.12 (0.6\%, n= 1), AY.4 (1.2\%, n= 2), AY.9 (1.2\%, n= 2) and Eta variant, B.1.525 $(0.6 \%, n=1) .11$ samples were not assigned any lineage.

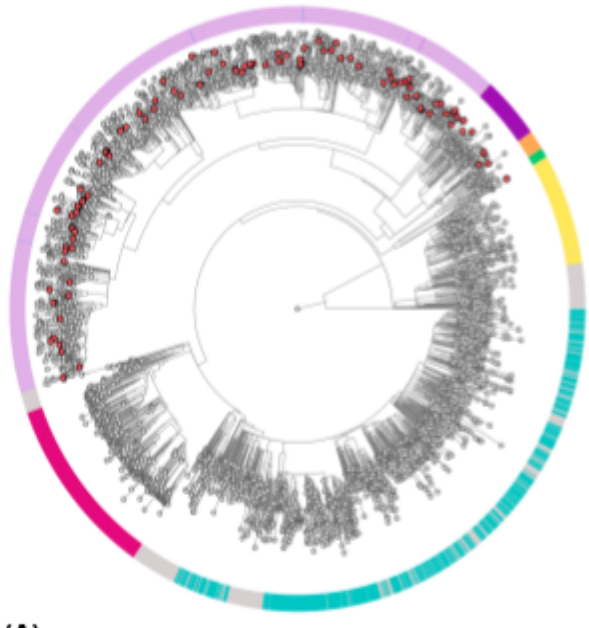

(A)

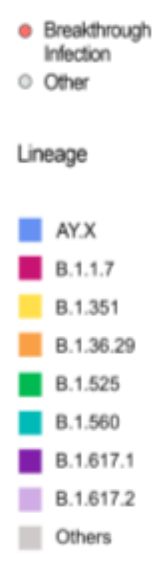

Others

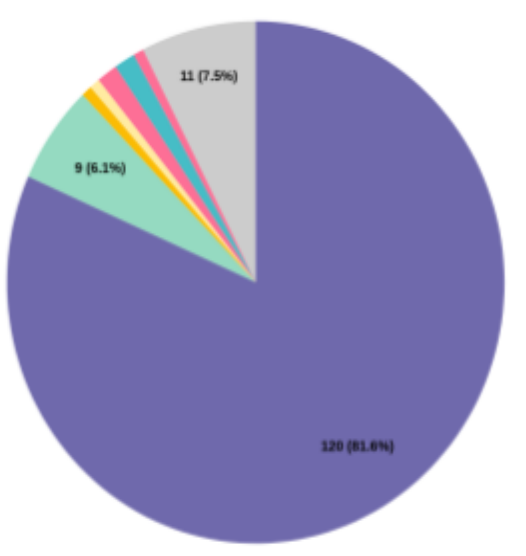

(B)
- B.1.617.2 $(n=120)$

- B.1.617.1 $(n=9)$

- AY.1 ( $n=1)$

AY.12 $(n=1)$

- AY.4 $(n=2)$

- AY.9 $(n=2)$

- B.1.525 ( $n=1)$

- No lineages ( $n=11$ )

Figure 1 (A)Phylogenetic context of breakthrough samples analysed (B) Summary of the distribution of lineages in breakthrough and control samples. 


\section{Discussion and Conclusion}

Mass vaccination efforts against COVID-19 are currently underway in many parts of the world as a mitigation strategy for the disease. However, the emergence of SARS-CoV-2 VoCs has raised concerns about the efficacy of the currently authorized vaccines against these variants. Current vaccines in use are reported to be safe with impressive efficacy rates against emerging variants of concern and interest. Phase III clinical trials report that ChAdOx1 nCoV-19 (Covishield) has a breakthrough infection rate of $0.2 \%$ to $0.6 \%{ }^{13}$ and BBV152/ (Covaxin) has a breakthrough rate of $0.77 \%$ in the cohort ${ }^{14,15}$. Recent reports have shown that even with lessened neutralisation of BBV152/Covaxin vaccinees, there is notable evidence that it confers protection against the Delta and Beta variant. Another study reveals that recipients of ChAdOx1 nCoV- 19 who have already been infected once and/or cases of breakthrough infection after vaccination have better chances of protection and immunity from the delta variant ${ }^{15}$.

In our study, all 147 patients were vaccinated with two doses of either AZD1222 or BBV152. A large proportion of VOC Delta (81.2\%) was found in the genome isolates analyzed, while the remaining isolates were also assigned as a VOC or VOI (Kappa and Eta). Analysis also showed that the occurrence of Delta in the vaccinated individuals was not significantly higher as compared to the prevalence of the Delta in the respective districts. From these findings we can conclude that despite the preponderance of the Delta variant in the breakthrough cases analysed, none of the cases had severe manifestations of the disease. Healthcare workers who are most likely to be adversely affected, presented only with amenable symptoms. Neutralisation responses are crucial in assessing vaccine efficacy and efficiency. Breakthrough infections must not be considered due to the inability of vaccines to confer immunity against an infection. It is plausible that newer lineages are a ramification of the ever evolving nature of viruses. Thus, deploying vaccines is crucial in bringing a pandemic under control.

\section{Funding}

Authors acknowledge the funding from National Health Mission Kerala and the Council of Scientific and Industrial Research (CSIR India) through grant MLP2005. BJ, MKD and AB acknowledge research fellowships from CSIR India. VG and SJA acknowledge research fellowships from University Grants Commission (UGC), India.

\section{Declaration of competing interest}

The authors declare no potential conflicts of interest.

\section{REFERENCES}

1. Skelly, D. T. et al. Two doses of SARS-CoV-2 vaccination induce more robust immune responses to 
emerging SARS-CoV-2 variants of concern than does natural infection. Research Square (2021) doi:10.21203/rs.3.rs-226857/v2.

2. McEwen, A. E. et al. Variants of Concern Are Overrepresented Among Postvaccination Breakthrough Infections of Severe Acute Respiratory Syndrome Coronavirus 2 (SARS-CoV-2) in Washington State. Clin. Infect. Dis. (2021) doi:10.1093/cid/ciab581.

3. Kustin, T. et al. Evidence for increased breakthrough rates of SARS-CoV-2 variants of concern in BNT162b2-mRNA-vaccinated individuals. Nat. Med. (2021) doi:10.1038/s41591-021-01413-7.

4. Duerr, R. et al. Dominance of Alpha and lota variants in SARS-CoV-2 vaccine breakthrough infections in New York City. doi:10.1101/2021.07.05.21259547.

5. Brinkley-Rubinstein, L., Peterson, M., Martin, R., Chan, P. \& Berk, J. Breakthrough SARS-CoV-2 Infections in Prison after Vaccination. N. Engl. J. Med. (2021) doi:10.1056/NEJMc2108479.

6. Lopez Bernal, J. et al. Effectiveness of the Pfizer-BioNTech and Oxford-AstraZeneca vaccines on covid-19 related symptoms, hospital admissions, and mortality in older adults in England: test negative case-control study. BMJ 373, n1088 (2021).

7. Wang, F. et al. Initial whole-genome sequencing and analysis of the host genetic contribution to COVID-19 severity and susceptibility. Cell Discovery 6, 1-16 (2020).

8. Cherian, S. et al. SARS-CoV-2 Spike Mutations, L452R, T478K, E484Q and P681R, in the Second Wave of COVID-19 in Maharashtra, India. Microorganisms 9, (2021).

9. Bhoyar, R. C. et al. High throughput detection and genetic epidemiology of SARS-CoV-2 using COVIDSeq next-generation sequencing. PLoS One 16, e0247115 (2021).

10. Wu, F. et al. A new coronavirus associated with human respiratory disease in China. Nature $\mathbf{5 7 9}$, 265-269 (2020).

11. Rambaut, A. et al. A dynamic nomenclature proposal for SARS-CoV-2 lineages to assist genomic epidemiology. Nature Microbiology 5, 1403-1407 (2020).

12. Yu, G. Using ggtree to Visualize Data on Tree-Like Structures. Curr. Protoc. Bioinformatics 69, (2020).

13. Safety and efficacy of the ChAdOx1 nCoV-19 vaccine (AZD1222) against SARS-CoV-2: an interim analysis of four randomised controlled trials in Brazil, South Africa, and the UK. Lancet 397, 99-111 
(2021).

14. Efficacy of ChAdOx1 nCoV-19 (AZD1222) vaccine against SARS-CoV-2 variant of concern 202012/01 (B.1.1.7): an exploratory analysis of a randomised controlled trial. Lancet 397, 1351-1362 (2021).

15. Ella, R. et al. Safety and immunogenicity of an inactivated SARS-CoV-2 vaccine, BBV152: interim results from a double-blind, randomised, multicentre, phase 2 trial, and 3-month follow-up of a double-blind, randomised phase 1 trial. Lancet Infect. Dis. 21, 950-961 (2021).

\section{Figure legends:}

Figure 1. (A)Phylogenetic context of breakthrough samples analysed

(B) Summary of the distribution of lineages in breakthrough and control samples.

\section{Supplementary Information}

1. Supplementary Table 1. Detailed list of samples recruited in the study. \# Kerala Breakthrough Sample list 